\title{
Prevenção de infecção de sítio cirúrgico em artroplastia: uma revisão das medidas recomendadas na prática
}

\section{Prevention of surgical site infection in arthroplasty: a review of the recommended measures in practice}

Taysa de Fátima Garcia ${ }^{1}$ • Adriana Cristina Oliveira

\begin{abstract}
RESUMO
Alnfecção de Sítio Cirúrgico (ISC) após cirurgias de artroplastia de quadril e joelho representam um sério problema de saúde, pois resultam em aumento dos custos e morbimortalidade dos pacientes acometidos. Objetiva-se identificar as medidas de prevenção de ISC em artroplastia de quadril e joelho descritas na literatura, e compará-las com as recomendações nacionais e internacionais. Conduziu-se uma revisão integrativa nas bases de dados da Biblioteca Virtual em Saúde (BVS), Cochrane, Pubmed, Science Direct e Scopus. Destacaram para prevenção da ISC, o uso profilático de antibióticos (76,0\%), o preparo cirúrgico da pele do paciente $(28,0 \%)$, banho com clorexidina $(24,0 \%)$, a descontaminação nasal $(12,0 \%)$, a paramentação cirúrgica e o uso de próteses com adição de antibióticos (16,0\%). Conclui-se que houve similaridade entre as medidas descritas nos estudos e aquelas recomendadas pelas diretrizes nacionais e internacionais. Não foram identificadas medidas como cuidado com a ferida operatória o que reforça a necessidade do olhar multiprofissional na prevenção de ISC.
\end{abstract}

Palavras-chave: Artroplastia do Quadril; Artroplastia do Joelho; Infecção Hospitalar.

\section{ABSTRACT}

The Surgical Site Infection (SSI) following hip and knee arthroplasty surgeries poses a severe health problem, resulting in an increase of both costs and morbimortality of inflicted patients. This study aimed to identifying SSI prevention measures in hip and knee arthroplasty described in literature, to compare them with the national and international guidelines recommendations. It was conducted an integrative review in databases of Biblioteca Virtual em Saúde (BVS), Cochrane, PubMed, Science Direct, and Scopus. These were highlighted for prevention of SSI: applications of prophylactic antibiotics $(76,0 \%)$, Skin preparation $(28,0 \%)$ chlorhexidine bath $(24,0 \%)$, nasal decontamination (12,0\%), Surgical paramentation and antibiotic-impregnated prosthesis $(16,0 \%)$. It concludes that there was a resemblance between the described measures on studies and those recommended by national and international guidelines. No measures were identified care for the surgical wound, which reinforces the need for multidisciplinary approach in SSI prevention.

Keywords: Arthroplasty Replacement Hip; Arthroplasty Replacement Knee; Cross Infection.

${ }^{1}$ Taysa de Fátima Garcia - Enfermeira. Mestre em Enfermagem pelo Programa de Pós-Graduação da Escola de Enfermagem da Universidade Federal de Minas Gerais. Membro do Núcleo de Pesquisa em Infecções Relacionadas ao Cuidas em Saúde/NEPIRCS. Belo Horizonte, Minas Gerais, Brasil. E-mail: taysafati@hotmail.com. Autor correspondente.

${ }^{2}$ Adriana Cristina Oliveira - Enfermeira. Mestre. Doutora em Enfermagem. Professora Associada da Escola de Enfermagem da Universidade Federal de Minas Gerais. Membro do Núcleo de Pesquisa em Infecções Relacionadas ao Cuidas em Saúde/NEPIRCS. Bolsista de Produtividade 1D CNPq. Belo Horizonte, Minas Gerais, Brasil. E-mail: adrianacoliveira@gmail.com. 


\section{INTRODUÇÃO}

As artroplastias de quadril e de joelho caracterizamse pela reconstrução ou substituição destas articulações por meio de próteses, seja total ou parcialmente. As artroplastias estão associadas a complicações graves, como a infecção incisional, superficial ou profunda de tecidos estéreis. Embora as taxas de infecção permaneçam entre $1 \%$ e $3 \%$, o número crescente de cirurgias de artroplastias de quadril e joelho aumenta a possibilidade de infecção. $\mathrm{Na}$ Inglaterra, anualmente, cerca de 2.500 artroplastias totais de quadril e 1.100 substituições de joelho são reabordadas devido à infecção cirúrgica ${ }^{1-2}$.

A Infecção do Sítio Cirúrgico (ISC) após artroplastia resulta em antibioticoterapia prolongada, aumento dos custos com tratamentos terapêuticos, podendo levar à novos procedimentos cirúrgicos como a revisão ou remoção de próteses. Nos Estados Unidos, o custo com cirurgias de revisões de artroplastias de quadril e joelho chegam a 566 milhões de dólares ao ano. No Reino Unido cerca de 1.594 euros são gastos a cada infecção cirúrgica levando à um custo anual de 1,5 a 19,1 bilhões de euros. A ocorrência de ISC impacta também no tempo médio de internação dos pacientes, prolongando sua permanência no hospital entre 2 a 49 dias $^{3-4}$.

Anualmente, o Centers for Disease Control and Prevention (CDC), por meio do National Healthcare Safety Network (NHSN), publica a incidência de ISC em diferentes procedimentos. No contexto das artroplastias de quadril e joelho, apresentaram taxas de infecção entre 2,6\% a 4,3\% em 2012, destacando-se dentre as infecções de sítio cirúrgico mais importantes. Em 2013, percebeu-se a redução dessas taxas em cerca de $27 \%$ a $40 \%$ respectivamente, e apesar de não atingir as metas estabelecidas pelo CDC, os dados demonstraram que as diretrizes para prevenção de ISC têm sido eficazes para a redução da infecção em várias partes do mundo ${ }^{1-5-6}$.

Recomendações para a prevenção da ISC são preconizadas em diretrizes específicas, e enfatizam medidas de acordo com cada fase do perioperatório, dentre elas: o banho pré-operatório do paciente com uso de antissépticos, a profilaxia antibiótica, tricotomia, preparação cirúrgica da pele, cuidados com a ferida operatória, paramentação cirúrgica e manutenção da homeostase do paciente no transoperatório, limpeza da sala cirúrgica e superfícies, entre outras ${ }^{7-9}$.

Especificamente sobre cirurgias ortopédicas, a Associação Britânica de Ortopedia (BOA) revisou em 2006 o Guideline "Primary total hip replacement: a guide to good practice", o qual refere além das medidas recomendadas pelo CDC, o uso profilático de antibióticos e implante de próteses cimentadas com adição de antibióticos ${ }^{10}$.

Mesmo com as recomendações publicadas em diversas partes do mundo, as ISC relacionadas a cirurgias ortopédicas com implante de próteses continuam a ocorrer significativamente, impactando seriamente na saúde pública. Diante da relevância deste tema questionou-se: as medidas para prevenção de infecção de sítio cirúrgico recomendadas pelas diretrizes nacionais e internacionais têm sido descritas para uma prática clínica mais segura?

Este estudo tem como objetivo identificar as medidas de prevenção de ISC em artroplastias de quadril e joelho descritas na literatura, e compará-las com as recomendações internacionais e nacionais.

\section{MÉTODO}

Este estudo consiste em uma revisão integrativa, a qual busca evidências científicas por meio da análise de múltiplos estudos, possibilitando a síntese do conhecimento e a determinação de lacunas do mesmo, proporcionando suporte na tomada de decisões ${ }^{11}$. Os artigos utilizados nesta revisão foram selecionados nas bases de dados da Biblioteca Virtual em Saúde (BVS), Biblioteca Cochrane, Pubmed, Science Direct e Scopus. Foram utilizados descritores controlados de acordo com a nomenclatura DeCS:Artroplastia de Quadril,Artroplastia de Joelho, Implante de Prótese. E descritores não controlados: Prevenção de Infecção em Artroplastia, Infecção da Ferida Operatória, Medidas Preventivas, Infecção de Prótese de Quadril, Infecção de Prótese de Joelho.

Os descritores foram pesquisados isoladamente na língua portuguesa e inglesa, posteriormente, foram realizadas associações entre eles, a fim de refinar a procura de artigos. Foram realizadas as seguintes associações: infecção de prótese de quadril AND medidas preventivas, infecção de prótese de joelho AND medidas preventivas, implante de prótese AND medidas preventivas, infecção de ferida operatória AND prevenção de infecção em artroplastia.

Foram delimitados artigos publicados a partir de 1999 , ano de publicação do "Guideline for Prevention of Surgical Site Infection" do CDC, que traz medidas específicas para a prevenção de infecção de sítio cirúrgico classificadas de acordo com o nível de evidência, ou seja, pela força científica, fundamentação teórica e aplicabilidade que cada uma representa.

As evidências são classificadas de acordo com o delineamento e condução do estudo que as originou, sendo apresentadas em níveis $1 \mathrm{~A}$ e $1 \mathrm{~B}$, que caracterizam medidas fortemente recomendadas, resultando de ensaios clínicos, experimentais, estudos epidemiológicos do tipo coorte e caso-controle bem delineados; nível de evidência Il consiste em estudos clínicos sugestivos, epidemiológicos e revisões sistemáticas bem delineadas. Existem ainda, aquelas medidas de prevenção que não possuem nível de evidência ou que não são devidamente esclarecidas, sendo classificadas como não resolvidas ${ }^{7}$.

Para verificação das práticas recomendadas foram revisadas as diretrizes e recomendações do Centers for Disease Control and Prevention's (CDC) “Guideline for Prevention of Surgical Site Infection" (1999)7; British Orthopaedic Association's (BOA) "Primary Total Hip 
Replacement: A Guide to Good Practice” (2006) ${ }^{10}$, National Institute of Clinical Excellence's (NICE) "Surgical Site Infection: Prevention and Treatment of Surgical Site Infection" $(2008)^{8} \mathrm{e}$ o National Health and Medical Research Council's (NHMRC) "Australian Guidelines for the Prevention and Control of Infection in Health Care" (2010) ${ }^{9}$. As medidas para prevenção da ISC, que nortearam a análise dos artigos selecionados, foram agrupadas e estão apresentadas no quadro 1.

Quadro 1. Medidas recomendadas pelos guidelines e diretrizes específicas para a prevenção da Infecção do Sítio Cirúrgico de acordo com seu grau de evidência.

\begin{tabular}{|c|c|}
\hline Medidas recomendadas & $\begin{array}{l}\text { Grau de evidência das } \\
\text { medidas de prevenção }\end{array}$ \\
\hline \multicolumn{2}{|c|}{ Pré-operatório } \\
\hline $\begin{array}{l}\text { Tricotomia (tricotomizador } \\
\text { elétrico) }\end{array}$ & $1 \mathrm{~A}^{1,2,3,4}$ \\
\hline $\begin{array}{l}\text { Descontaminação nasal em } \\
\text { pacientes colonizados }\end{array}$ & $1 \mathrm{~B}^{2}$ \\
\hline $\begin{array}{l}\text { Banho pré-operatório com } \\
\text { clorexidina }\end{array}$ & $1 \mathrm{~B}^{1,2,3,4}$ \\
\hline $\begin{array}{l}\text { Esterilização do instrumental } \\
\text { cirúrgico }\end{array}$ & $1 \mathrm{~B} 1,2,3,4$ \\
\hline $\begin{array}{l}\text { Limpeza do ambiente e } \\
\text { superfícies }\end{array}$ & $1 \mathrm{~B}^{1,2}$ \\
\hline Profilaxia com antibióticos & $1 \mathrm{~A}^{1,2,3,4}$ \\
\hline $\begin{array}{l}\text { Preparação antisséptica da pele } \\
\text { do paciente }\end{array}$ & $1 \mathrm{~B}^{1,2,3,4}$ \\
\hline $\begin{array}{l}\text { Preparo cirúrgico das mãos do } \\
\text { cirurgião }\end{array}$ & $\mathrm{IB}^{1,2,3,4}$ \\
\hline Redução do tempo de internação & $\|^{1}$ \\
\hline \multicolumn{2}{|c|}{ Transoperatório } \\
\hline Climatização da sala operatória & $1 \mathrm{~B}^{1,2}$ \\
\hline Uso duplo de luvas estéreis & $1 \mathrm{~B}^{1,2,3,4}$ \\
\hline Paramentação cirúrgica & $1 \mathrm{~B}^{1,2,3,4}$ \\
\hline Homeostase do paciente & $1 \mathrm{~B}^{1,3}$ \\
\hline Tempo de duração da cirurgia & Não resolvido \\
\hline $\begin{array}{l}\text { Uso de drenos fechados na } \\
\text { incisão cirúrgica }\end{array}$ & $1 \mathrm{~B}^{1}$ \\
\hline $\begin{array}{l}\text { Uso de prótese cimentada com } \\
\text { adição de antibióticos }\end{array}$ & Não resolvido \\
\hline \multicolumn{2}{|l|}{ Pós-operatório } \\
\hline $\begin{array}{l}\text { Orientação aos pacientes no pós- } \\
\text { operatório }\end{array}$ & $\|^{1}$ \\
\hline $\begin{array}{l}\text { Curativo estéril da ferida por } 24 \text { a } \\
48 \text { horas após a cirurgia }\end{array}$ & $1 \mathrm{~B}^{1,2,3,4}$ \\
\hline $\begin{array}{l}\text { Vigilância dos pacientes após a } \\
\text { alta hospitalar }\end{array}$ & $1 \mathrm{~B}^{1}$ \\
\hline \multicolumn{2}{|c|}{$\begin{array}{l}\text { Fonte: dados da pesquisa. } \\
\text { 1. CDC - Guideline for Prevention of Surgical Site Infection (1999) } \\
\text { 2. NHMRC - Australian Guidelines for the Prevention and Control o } \\
\text { Infection in Health Care (2010) } \\
\text { 3. NICE - Surgical Site Infection: Prevention and Treatment of Surgica } \\
\text { Site Infection (2008) }\end{array}$} \\
\hline \multicolumn{2}{|c|}{ 4. BOA - Primary Total Hip Replacement: A Guide to Good Practice (2006 } \\
\hline
\end{tabular}

Delimitou-se como critérios de inclusão: estudos originais, publicados na íntegra, disponíveis gratuitamente, sem restrição de idiomas, publicados entre 1999 a
2015, e que abordassem as medidas de prevenção da ISC em artroplastias de quadril e joelho; nos diferentes momentos do perioperatório. Pesquisas que abordaram especificamente a técnica cirúrgica, bem como aqueles referentes à prevenção de tromboembolia venosa foram excluídos do estudo.

Os artigos foram analisados quanto às medidas adotadas para prevenção de ISC, em artroplastias de quadril e joelho, em conformidade com o recomendado pelas diretrizes específicas; quanto à frequência em que foram descritas nos artigos, e quanto ao nível de evidência do estudo. A análise foi baseada na categorização da Agency for Healthcare Research and Quality (AHRO) dos EUA ${ }^{12}$, que classifica os estudos conforme a abordagem metodológica adotada, em seis níveis, sendo: 1: evidências resultantes da metaanálise (estudos clínicos controlados e randomizados); 2 : obtidas de estudos com experimentais; 3: evidências de estudos quase experimentais; 4: estudos descritivos (não experimentais) ou qualitativos; 5 : resultados provenientes de relatos de caso/experiência; e 6: evidências baseadas em opiniões ou consensos de especialistas. Os resultados foram representados por meio de gráficos e tabelas.

\section{RESULTADOS}

Foram selecionados 67 artigos, por meio de leitura exploratória do resumo. Aqueles que após leitura analítica, não atenderam aos critérios de inclusão foram excluídos, delimitando-se a 25 artigos. A relação entre ano, país e nível de evidência dos artigos está descrita no quadro 2.

Para contrastar com as diretrizes recomendadas, foram identificadas as principais medidas, descritas nos artigos selecionados, para cada fase do perioperatório. As práticas que destacaram-se para prevenção de ISC em artroplastias no período pré-operatório foram: a profilaxia com antibióticos descrita em 76,0\% dos estudos; o banho préoperatório com clorexidina $24,0 \%$; o preparo antisséptico da pele do paciente $28,0 \%$ e descontaminação nasal com mupirocina $12,0 \%$.

No transoperatório, foram predominantemente citados: o uso de próteses cimentadas com adição de antibióticos e a paramentação cirúrgica em $16,0 \%$, respectivamente, a climatização da sala operatória e o uso de drenos fechados na incisão cirúrgica em $8,0 \%$ dos artigos. Dentre as práticas encontradas, referentes ao pós-operatório, percebeu-se a vigilância pós alta em $8,0 \%$ dos estudos. As medidas de prevenção, de acordo com cada guideine/diretriz que as apresenta, estão dispostas na tabela 1 , a seguir.

As medidas descritas foram analisadas quanto à frequência em que apareceram nos estudos, e separadas por período operatório. Gráfico 1.

Percebeu-se a predominância de profissionais médicos na autoria dos artigos selecionados, $100 \%$ dos estudos apresentaram profissionais médicos como autores. 
Quadro 2. Relação entre ano, país e nível de evidencia dos artigos analisados, 1999 - 2015.

\begin{tabular}{|c|c|c|c|c|}
\hline Título & Autor & Ano & País & $\begin{array}{l}\text { Nível de } \\
\text { evidência }\end{array}$ \\
\hline $\begin{array}{l}\text { Efficacy of A Single Dose of Cefazolin as a Prophylactic Antibiotic in } \\
\text { Primary Arthroplasty }\end{array}$ & Tang et al. ${ }^{13}$ & 2003 & China & 3 \\
\hline $\begin{array}{l}\text { Évaluation du risque infectieux sur prothèses totales de hanche et de } \\
\text { genou: À propos d'une série continue de } 1000 \text { prothèses }\end{array}$ & Eveillard et al. ${ }^{14}$ & 2005 & França & 3 \\
\hline $\begin{array}{l}\text { Outcome following deep wound contamination in cemented } \\
\text { arthroplasty }\end{array}$ & Byrne et al. ${ }^{15}$ & 2006 & Irlanda & 3 \\
\hline $\begin{array}{l}\text { Surgical site infection after total knee arthroplasty: a monocenter } \\
\text { analysis of } 923 \text { first-intention implantations }\end{array}$ & Debarge et al. ${ }^{16}$ & 2007 & França & 3 \\
\hline $\begin{array}{l}\text { Efecto de la vigilancia sobre la tasa de infección de la herida quirúrgica } \\
\text { en prótesis de cadera y rodilla }\end{array}$ & Cabrillana et al. ${ }^{17}$ & 2007 & Espanha & 3 \\
\hline $\begin{array}{l}\text { Staphylococcus aureus Nasal Decolonization in Joint Replacement } \\
\text { Surgery Reduces Infection }\end{array}$ & Hacek et al. ${ }^{18}$ & 2008 & EUA & 3 \\
\hline $\begin{array}{l}\text { Adherence to recommendations designed to decrease intra-operative } \\
\text { wound contamination }\end{array}$ & $\begin{array}{l}\text { Mackain-Bremner } \\
\text { et al. }{ }^{19}\end{array}$ & 2008 & $\begin{array}{l}\text { Reino } \\
\text { Unido }\end{array}$ & 4 \\
\hline $\begin{array}{l}\text { Effect of Procedure Duration on Total Hip Arthroplasty and Total Knee } \\
\text { Arthroplasty Survivorship in the United States Medicare Population }\end{array}$ & Ong et al. ${ }^{20}$ & 2008 & EUA & 4 \\
\hline Antibiotic Prophylaxis in Primary Hip and Knee Arthroplasty & Tyllianakis et al. ${ }^{21}$ & 2010 & Grécia & 2 \\
\hline Infection risk prevention following total knee arthroplasty & Levent et al. ${ }^{22}$ & 2010 & França & 3 \\
\hline $\begin{array}{l}\text { Current Practice Patterns in Primary Hip and Knee Arthroplasty Among } \\
\text { Members of the American Association of Hip and Knee Surgeons }\end{array}$ & Berry, Bozic. ${ }^{23}$ & 2010 & EUA & 4 \\
\hline $\begin{array}{l}\text { Preoperative Chlorhexidine Preparation and the Incidence of Surgical } \\
\text { Site Infections After Hip Arthroplasty }\end{array}$ & Johnson et al. ${ }^{24}$ & 2010 & EUA & 3 \\
\hline $\begin{array}{l}\text { Preoperative Screening/Decolonization for Staphylococcus aureus to } \\
\text { Prevent Orthopedic Surgical Site Infection }\end{array}$ & Rao et al. ${ }^{25}$ & 2011 & EUA & 3 \\
\hline $\begin{array}{l}\text { Incidence and associated factors of surgical site infections after hip } \\
\text { arthroplasty }\end{array}$ & Vélez et al. ${ }^{26}$ & 2011 & Itália & 3 \\
\hline $\begin{array}{l}\text { Advance pre-operative chlorhexidine reduces the incidence of surgical } \\
\text { site infections in knee arthroplasty }\end{array}$ & Zywiel et al. ${ }^{27}$ & 2011 & EUA & 3 \\
\hline $\begin{array}{l}\text { Is it Time to Include Vancomycin for Routine Perioperative Antibiotic } \\
\text { Prophylaxis in Total Joint Arthroplasty Patients? }\end{array}$ & Smith et al. ${ }^{28}$ & 2012 & EUA & 3 \\
\hline $\begin{array}{l}\text { Cefuroxime-impregnated cement and systemic cefazolin for } 1 \text { week in } \\
\text { primary total knee arthroplasty: An evaluation of } 2700 \text { knees }\end{array}$ & Chiang, Chiu. ${ }^{29}$ & 2012 & China & 3 \\
\hline $\begin{array}{l}\text { Does Dual Antibiotic Prophylaxis Better Prevent Surgical Site Infections } \\
\text { in Total Joint Arthroplasty? }\end{array}$ & Sewick et al. ${ }^{30}$ & 2012 & EUA & 3 \\
\hline $\begin{array}{l}\text { An evaluation of immediate-use steam sterilization practices in adult } \\
\text { knee and hip arthroplasty procedures }\end{array}$ & Zuckerman et al. ${ }^{31}$ & 2012 & EUA & 2 \\
\hline $\begin{array}{l}\text { Epidemiologia da artroplastia total de quadril e de joelho: estudo } \\
\text { transversal }\end{array}$ & Lenza et al. ${ }^{32}$ & 2013 & Brasil & 4 \\
\hline $\begin{array}{l}\text { Advance pre-operative chlorhexidine preparation reduces periprosthetic } \\
\text { infections following total joint arthroplasty }\end{array}$ & Kapadia et al. ${ }^{33}$ & 2013 & EUA & 3 \\
\hline $\begin{array}{l}\text { Bacterial contamination of the wound during primary total hip and } \\
\text { knee replacement Median } 13 \text { years of follow-up of } 90 \text { replacements }\end{array}$ & Jonsson et al. ${ }^{34}$ & 2014 & Islândia & 4 \\
\hline $\begin{array}{l}\text { Risk Stratified Usage of Antibiotic-Loaded Bone Cement for Primary } \\
\text { Total Knee Arthroplasty: Short Term Infection Outcomes With a } \\
\text { Standardized Cement Protocol }\end{array}$ & Qadir et al. ${ }^{35}$ & 2014 & EUA & 4 \\
\hline $\begin{array}{l}\text { Is Preoperative Staphylococcal Decolonization Efficient in Total Joint } \\
\text { Arthroplasty }\end{array}$ & Moroski et al. ${ }^{36}$ & 2015 & EUA & 3 \\
\hline $\begin{array}{l}\text { Alcoholic Chlorhexidine or Alcoholic lodine Skin Antisepsis (ACAISA): } \\
\text { protocol for cluster randomised controlled trial of surgical skin } \\
\text { preparation for the prevention of superficial wound complications in } \\
\text { prosthetic hip and knee replacement surgery }\end{array}$ & Peel et al. ${ }^{37}$ & 2015 & Austrália & 2 \\
\hline
\end{tabular}

Fonte: dados da pesquisa. 
Tabela 1. Medidas Recomendadas em cada guideline/diretriz e frequência descritas nos estudos analisados, 1999 a 2015.

\begin{tabular}{|c|c|c|c|}
\hline & Medidas recomendadas & Guideline/diretrizes & Frequência descrita nos artigos (\%) \\
\hline \multirow{9}{*}{ 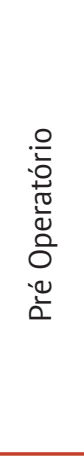 } & Tricotomia (tricotomizador elétrico) & CDC/NICE/NHMRC/BOA & $4,0 \%{ }^{(15)}$ \\
\hline & $\begin{array}{c}\text { Descontaminação nasal em pacientes } \\
\text { colonizados }\end{array}$ & NICE/NHMRC & $12,0 \%(18,25,36)$ \\
\hline & Banho pré-operatório com clorexidina & CDC/NICE/NHMRC & $24,0 \%(15,22,24,33,35,36)$ \\
\hline & Esterilização do instrumental cirúrgico & CDC/NICE/NHMRC & $4,0 \%(31)$ \\
\hline & Limpeza do ambiente e superfícies & CDC/BOA & $4,0 \%{ }^{(22)}$ \\
\hline & Profilaxia com antibióticos & CDC/NICE/NHMRC/BOA & $76,0 \%{ }^{(13-19,21-23,25,26,28-30,32,34-36)}$ \\
\hline & Preparação antisséptica da pele do paciente & CDC/NICE/NHMRC/BOA & $28,0 \%(15,21,22,27,34,35,37)$ \\
\hline & Preparo cirúrgico das mãos do cirurgião & CDC/NICE/NHMRC/BOA & - \\
\hline & Redução do tempo de internação & $\mathrm{CDC}$ & $4,0 \%(26)$ \\
\hline \multirow{7}{*}{ 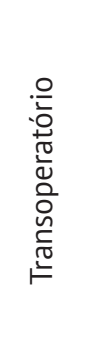 } & Climatização da sala operatória & CDC/BOA/NHMRC & $8,0 \%{ }^{(22,34)}$ \\
\hline & Uso duplo de luvas estéreis & CDC/NICE/NHMRC/BOA & - \\
\hline & Paramentação cirúrgica & CDC/NICE/NHMRC & $16,0 \%^{(15,19,22,34)}$ \\
\hline & Homeostase do paciente & CDC/NICE & - \\
\hline & Tempo de duração da cirurgia & $\mathrm{BOA}$ & $4,0 \%(20)$ \\
\hline & Uso de drenos fechados na incisão cirúrgica & CDC & $8,0 \%(23,32)$ \\
\hline & $\begin{array}{l}\text { Uso de prótese cimentada com adição de } \\
\text { antibióticos }\end{array}$ & $\mathrm{BOA}$ & $16,0 \%(14,15,29,35)$ \\
\hline \multirow{3}{*}{ 岁总 } & Orientação aos pacientes no pós-operatório & CDC & - \\
\hline & $\begin{array}{l}\text { Curativo estéril da ferida por } 24 \text { a } 48 \text { horas } \\
\text { após a cirurgia }\end{array}$ & CDC/NICE/NHMRC/BOA & - \\
\hline & $\begin{array}{c}\text { Vigilância dos pacientes após a alta } \\
\text { hospitalar }\end{array}$ & CDC & $8,0 \%(17,32)$ \\
\hline
\end{tabular}

Fonte: dados da pesquisa.

CDC - Guideline for Prevention of Surgical Site Infection (1999)

NHMRC - Australian Guidelines for the Prevention and Control of Infection in Health Care (2010)

NICE - Surgical Site Infection: Prevention and Treatment of Surgical Site Infection (2008)

BOA - Primary Total Hip Replacement: A Guide to Good Practice (2006)

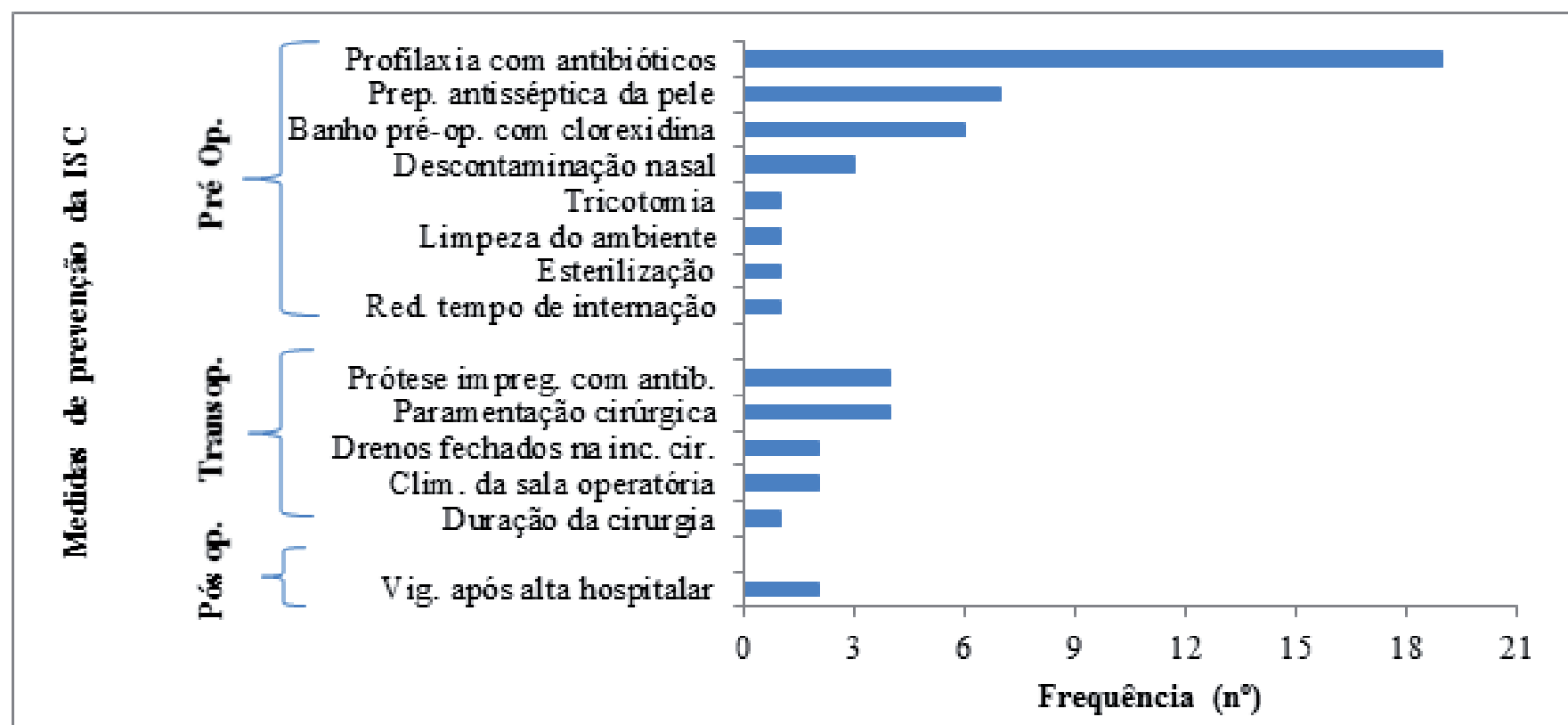

Gráfico 1: Frequência das medidas descritas nos estudos em cada fase do perioperatório, 1999 a 2015.

Fonte: dados da pesquisa.

\section{DISCUSSÃO}

A ISC é um importante problema de saúde, e a adesão às medidas para sua prevenção deve ser assegurada pelos serviços que prestam assistência ao paciente cirúrgico. Embora normas específicas para prevenção de ISC sejam recomendadas em diferentes diretrizes, estima-se que a ocorrência deste evento decorre da lacuna no cumprimento sistemático das medidas preventivas pela equipe cirúrgica, nas falhas de interpretação das medidas e na falta de recursos ${ }^{7-4-38}$. 
A internação pré-operatória prolongada aumenta a possibilidade de colonização bacteriana do paciente cirúrgico, portanto é sensato que pacientes submetidos à cirurgias de artroplastias sejam internados no máximo até o dia anterior ou no mínimo no dia da cirurgia ${ }^{10}$. Portanto, alguns fatores são determinantes da internação acima do período recomendado para pacientes submetidos à cirurgias ortopédicas, como: a idade; a presença de comorbidades que necessitam ser tratadas no pré-operatório; a instabilidade hemodinâmica, como níveis de hemoglobina alterados; e a necessidade de hemotransfusão antes da cirurgia ${ }^{39-40}$.

Um estudo publicado em $2014^{41}$ identificou a incidência de ISC de $14 \%$ em pacientes internados previamente (média de 7,6 dias), índice significativamente maior quando comparados àqueles que internaram no dia da operação, com incidência de $3,2 \%$. Outra pesquisa realizada nos EUA em $2012^{42}$, corrobora em seu estudo, com resultado de taxas de infecção entre $3,1 \%$ a $4,3 \%$ nos pacientes com internação prévia entre 5 a 8 dias.

Outro fator importante relacionado à possibilidade de desenvolvimento de ISC diz respeito à duração da cirurgia. Neste mesmo estudo ${ }^{42}$, foi demonstrado que cirurgias com duração entre 150 a 249 minutos apresentaram taxas de infecção cirúrgica entre $9 \%$ a 13,9\%, quando comparadas à procedimentos de curta duração, menor que 150 minutos. Resultado semelhante é retratado em outro estudo ${ }^{20}$, o qual demonstrou que artroplastias de joelho e quadril com duração entre 150 a 180 minutos apresentam maior possibilidade de revisão cirúrgica além de aumentar o risco de hemorragia e tromboembolia venosa ${ }^{42-20}$.

O tempo de exposição da cavidade cirúrgica favorece contaminação da ferida operatória devido à fatores como a suspensão de partículas na sala de operação, estas que podem servir como veículo para que microrganismos patogênicos entrem em contato com o local da cirurgia, contaminando-a ${ }^{20-43}$. 0 uso de prótese cimentada com adição de antibióticos é recomendado pela Associação Britânica de Ortopedia para artroplastias primárias ou revisões ${ }^{10}$. Embora seu uso seja controverso, um estudo realizado na Noruega ${ }^{44}$, identificou que a incidência de revisão em artroplastia, decorrente de ISC, foi menor em cirurgias que utilizaram prótese cimentada com antibiótico em comparação ao uso do cimento comum.

Entretanto, outro estudo que avaliou o uso rotineiro de cimento ósseo impregnado com antibióticos ${ }^{45}$, demonstrou que as taxas reduzidas de infecção após artroplastia de quadril cimentada com antibióticos não foram significativamente diferentes das que utilizaram cimento regular não impregnado. 0 uso de próteses com adição de antibióticos, além de não apresentar eficácia devidamente comprovada para prevenção de ISC, contribui com o desenvolvimento da resistência bacteriana. Estimase que cerca de $8 \%$ do antibiótico utilizado em cimentos ortopédicos é liberado imediatamente após a cirurgia, posteriormente, a liberação torna-se lenta e as doses baixas, insuficientes para combater as infecções, sendo favoráveis para o desenvolvimento de cepas resistentes, tornando o tratamento ainda mais difícil ${ }^{45}$.

Embora as medidas descritas nos artigos selecionados corroborem com as diretrizes, recomendações como higienização e preparo cirúrgico das mãos, e cuidados com a ferida operatória, não foram descritas nos estudos analisados; enquanto o uso de antibióticos foi amplamente mencionado. A profilaxia antibiótica reduz a carga bacteriana presente no local da incisão cirúrgica diminuindo as chances de ocorrência de ISC, todavia não é uma medida independente. A contaminação do local da cirurgia e o possível desenvolvimento de infecção podem decorrer da presença de bactérias em instrumentais cirúrgicos, na pele do paciente, nas mãos dos profissionais, ou por falhas no cuidado com a ferida operatória após a cirurgia ${ }^{46-47}$.

Ressalta-se, portanto, que a ocorrência da ISC é multifatorial e exige a colaboração e adesão multiprofissional às medidas preventivas de infecção, objetivando implementá-las conforme recomendado, em cada fase do perioperatório, reforçando a importância de cada uma delas no processo de prevenção de infecção cirúrgica ${ }^{38}$. A atuação multiprofissional na prevenção da infecção de sítio cirúrgico é fundamental, especialmente por descentralizar a atuação apenas do profissional médico na prescrição de medidas farmacológicas como o uso de antibióticos profiláticos, clorexidina no banho préoperatório e mupirocina para descolonização nasal.

O trabalho de cada profissional envolvido na assistência ao paciente cirúrgico é de extrema importância para a prevenção de ISC uma vez que esta pode decorrer de um curativo inadequado no pós-operatório, da tricotomia realizada incorretamente no pré-operatório ou de falhas nos processos de esterilização do instrumental cirúrgico. Medidas como estas são recomendadas e enfatizam a ocorrência multifatorial da ISC, o que reforça a atuação de médicos, enfermeiros e técnicos de enfermagem na sua prevenção e segurança do paciente cirúrgico.

\section{CONCLUSÃO}

Embora o uso profilático de antibióticos em cirurgias ortopédicas represente adesão significativamente superior às demais medidas recomendadas, o seu uso individual não é suficiente para prevenir a ISC. Para que o cumprimento destas medidas ocorra de forma eficiente, é necessária a atuação de todos os envolvidos na assistência ao paciente cirúrgico. A abordagem criteriosa das recomendações devem ser complementares e desenvolvidas em conjunto, garantindo o cumprimento e a realização de cada uma delas no pré-operatório, intraoperatório e pós-operatório.

Percebeu-se que as medidas de prevenção da ISC e sua importância em cada fase do perioperatório foram pouco exploradas nos artigos selecionados, apontando notória deficiência no tocante à investigação e pesquisa acerca das medidas de prevenção da ISC. Ressalta-se que 
este tema representa relevância e importância para toda a equipe cirúrgica, especialmente à equipe de enfermagem.

\section{REFERÊNCIAS}

1. Urquhart DM. et al. Incidence and risk factors for deep surgical site infection after primary total hip arthroplasty: A systematic review. J Arthroplasty. [Internet]. 2010 [acesso em: $10 \mathrm{dez}$ 2015]; 25(8):1216-22. Disponível em: https://www.ncbi.nlm. nih.gov/pubmed/19879720.

2. Malhas AM, Lawton R, Reidy M, Nathwani D, Clift BA. Causative organisms in revision total hip \& knee arthroplasty for infection: Increasing multiantibiotic resistance in coagulasenegative Staphylococcus and the implications for antibiotic prophylaxis. Surge-on. [Internet]. 2014 [acesso em: 23 jul 2015]; (6):1-6. Disponível em: https://www.ncbi.nlm.nih.gov/ pubmed/24821264.

3. Kapadia BH, McElroy MJ, Issa K, Jonhson AJ, Bozic KJ, Mont MA. The Econom-ic Impact of Periprosthetic Infections Following Total Knee Arthroplasty at a Specialized Tertiary-Care Centers. J Arthroplasty. [Internet]. 2014 [acesso em: 05 abr 2015]; 29:929-32. Disponível em: https://www.ncbi.nlm.nih.gov/ pubmed/24140271.

4. OMS. Organização Mundial da Saúde. Aliança mundial para segurança do paciente. Cirurgias Seguras Salvam Vidas. OMS. [Internet]. 2008. [acesso em: 20 jul 2015]. Disponí-vel em: https://www.into.saude.gov.br/upload/arquivos/pacientes/ cirurgias_seguras/Seguran\%C3\%A7a_do_Paciente_guia.pdf.

5. CDC. Centers for Diseases Control and Prevention. National and State Healthcare Associeted Infections. Progress Report. CDC. [Internet]. 2014. [acesso em: 06 jun 2015]. Disponível em: https://www.cdc.gov/hai/surveillance/progress-report/

6. CDC. Centers for Diseases Control and Prevention. National and State Healthcare Associeted Infections. Progress Report. This report is based on 2013 data, published janu-ary 2015. CDC. [Internet]. 2015. [acesso em: 10 jan 2016]. Disponível em: https://www.cdc.gov/hai/surveillance/progress-report/.

7. CDC. Centers for Disease Control and Prevention. Guideline for Prevention of Surgical Site Infection, 1999. CDC. [Internet]. 1999. [acesso em: 16 mai 2015]. Disponível em: https://www. cdc.gov/infectioncontrol/guidelines/index.html

8. NICE. National Institute for Health and Clinical Excellence. Surgical site infection prevention and treatment of surgical site infection. NICE. [Internet]. 2008. [acesso em: 19 mai 2015]. Disponível em: https://www.nice.org.uk/guidance/cg74.

9. NHMRC. National Health and Medical Research Council. Australian Comission on Safety and Quality in Healthcare. Australian guidelines for the prevention and control of infection in healthcare. NHMRC. [Internet]. 2010. [acesso em: 19 mai 2015]. Disponível em: https://www.nhmrc.gov.au/ guidelines-publications/cd33.

10. BOA. Brithsh Orthopaedic Association. Primary Total Hip Replacement: A guide to good practice. BOA. [Internet]. 2006. [acesso em: 24 mai 2015]. Disponível em: https://www.boa. ac.uk/pro-practice/guidance-and-good-practice/.

11. Mendes KDS, Silveira RCCP, Galvão CM. Revisão integrativa: método de pesquisa para incorporação de evidências na saúde e na enfermagem. Texto e Contexto Enferma-gem. [Internet]. 2008. [acesso em: 03 ago 2015];17(4): 758-64.
Disponível em: http://www.scielo.br/scielo.php?pid=S0104$07072008000400018 \&$ script=sci_abstract $\&$ tlng=pt.

12. Stetler CB, Morsi D, Rucki S, Broughton S, Corrigan B, Fitzgerald $J$ et al. Utilization fo-cused integrative reviews in a nursing service. Appl Nurs Res. [Internet]. 1998. [acesso em: 24 mai 2015]; 11(4):195-206. Disponível em: https://www.ncbi.nlm. nih.gov/pubmed/?term=Utilization+focused+integrative+ reviews+in+a+nursing+service.

13. Tang WM, Chiu KY, Ng TP, Yau WP, Ching PTY, Seto WH. Efficacy of A Single Dose of Cefazolin as a Prophylactic Antibiotic in Primary Arthroplasty. J Arthroplast. [In-ternet]. 2003. [acesso em: 30 jun 2015]; 18: 714-718. Disponível em: https://www. ncbi.nlm.nih.gov/pubmed/14513443.

14. Eveillard M, Canarelli B, Lavenne J, Eb F, Mertl P. Évaluation du risque infectieux sur prothèses totales de hanche et de genou $A$ propos d'une série continue de 1000 pro-thèses.Antibiotiques. [Internet]. 2005. [acesso em: 3 ago 2015]; 7: 87-92. Disponível em: https://www.researchgate.net/publication/257515341_ Evaluation_du_risque_infectieux_sur_protheses_totales_de hanche_et_de_genou_A_propos_d\%27une_serie_continue_ de_1_000_protheses.

15. Byrne AM, Morris S, McCarthy, Quinlan W, O'Byrne JM. Outcome following deep wound contamination in cemented arthroplasty. Int Orthop. [Internet]. 2007. [acesso em: 20 jun 2015]; 31: 27-31. Disponível em: https://www.ncbi.nlm.nih. gov/pmc/articles/PMC2267550/.

16. Debarge R, Nicolle MC, Pinaroli A, Selmi TAS, Neyret P. Infection du site opéra-toire après arthroplastie totale de genou Taux observé après 923 interventions dans une cen-tre formateur. Revue de chirurgie orthopédique. [Internet]. 2007. [acesso em: 11 ago 2015]; 93: 582-87. Disponível em: http:// www.em-consulte.com/en/article/132008.

17. Cabrillana JM, et al. Efecto de la vigilancia sobre la tasa de infección de la herida quirúrgica en prótesis de cadera y rodilla. Rev Clin Esp. [Internet]. 2007. [acesso em: 11 ago 2015];207(8):388-93. Disponível em: http://www. revclinesp.es/es/efecto-vigilancia-sobre-tasa-infeccion/ articulo/13108756/.

18. Hacek MD, Robb WJ, Paule SM, Kudrna JC, Stamos VP, Peterson LR. Staphylo-coccus aureus Nasal Decolonization in Joint Replacement Surgery Reduces Infection. Clin Orthop Relat Res. [Internet]. 2008. [acesso em: 10 ago 2015]; 466:1349-55. Disponível em: https://www.ncbi.nlm.nih.gov/ pubmed/18347889.

19. Mackain-Bremner AA, Owens K, Wylde V, Bannister GC, Blom AW. Adherence to recommendations designed to decrease intra-operative wound contamination. Ann R Coll Surg Engl. [Internet]. 2008. [acesso em: 16 ago 2015]; 90: 412-16. Disponível em: https://www.ncbi.nlm.nih.gov/pmc/articles/ PMC2645751/.

20. Ong KL, Lau E, Manley M, Kurtz SM. Effect of Procedure Duration on Total Hip Arthroplasty and Total Knee Arthroplasty Survivorship in the United States Medicare Population. J Arthroplasty. [Internet]. 2008. [acesso em: 27 jun 2015] Sep; 23(6): 127-32. Disponível em: https://www.ncbi.nlm.nih.gov/ pubmed/18555641.

21. Tyllianakis ME, Karageorgos AC, Marangos MN, Sarids AG, Lambiris EE. Antibi-otic Prophylaxis in Primary Hip and Knee Arthroplasty. Comparison Between Cefuroxime and Two 
Specific Antistaphylococcal Agents. J Arthroplasty. [Internet] 2010. [acesso em: 13 ago 2015]; 25(7): 1078-82. Disponível em: https://www.ncbi.nlm.nih.gov/pubmed/20381287.

22. Levent T, et al. Infection risk prevention following total knee arthroplasty. Orthop Traumatol Surg Res. [Internet]. 2010. [acesso em: 21 ago 2015]; 96: 49-56. Disponível em: https:// www.ncbi.nlm.nih.gov/pubmed/20170857.

23. Berry D, Bozic KJ. Current Practice Patterns in Primary Hip and Knee Arthroplasty Among Members of the American Association of Hip and Knee Surgeons.J Arthroplasty. [Internet]. 2010. [acesso em: 20 ago 2015]; 25 (6): 2-4. Disponível em: https://www.ncbi.nlm.nih.gov/pubmed/20580196.

24. Johnson AJ, Daley JA, Zywiel MG, Delanois RE, Mont MA. Preoperative Chlor-hexidine Preparation and the Incidence of Surgical Site Infections After Hip Arthroplasty. J Arthroplasty. [Internet]. 2010. [acesso em: 5 set 2015]; 25(6): 98-102. Disponível em: https://www.ncbi.nlm.nih.gov/ pubmed/20570089.

25. Rao N, Cannella B, Crossett LS, Yates JAJ, McGough RL, Hamilton CW. Preoper-ative Screening/Decolonization for Staphylococcus aureus to Prevent Orthopedic Surgical Site Infection Prospective Cohort Study With 2-Year Follow-Up. J Arthroplasty. [Inter-net]. 2011. [acesso em: 21 ago 2015]; 26(8): 1501-07. Disponível em: https://www.ncbi.nlm.nih.gov/ pubmed/21507604.

26. Vélez AEG, Pérez DA, Rodela AR, Jodrá VM. Incidence and associated factors of surgical site infections after hip arthroplasty. Rev Esp Cir Ortop Traumatol. [Internet]. 2011. [acesso em: 25 ago 2015]; 4(55): 270-76. Disponível em: http://www.elsevier.es/es-revista-revista-espanolacirugia-ortopedica-traumatologia-429-articulo-incidenceassociated-factors-surgical-site-S1988885611703182.

27. Zywiel MG, Daley JA, Delanois RE, Naziri Q, Johnson AJ, Mont MA. Advance pre-operative chlorhexidine reduces the incidence of surgical site infections in knee arthro-plasty. Int Orthop. [Internet]. 2011. [acesso em: 05 set 2015]; 35: 1001-06. Disponível em: https://www.ncbi.nlm.nih.gov/ pubmed/20563806.

28. Smith EB, Wynne R, Joshi A, Liu H, Good RP. Is it Time to Include Vancomycin for Routine Perioperative Antibiotic Prophylaxis in Total Joint Arthroplasty Patients?. J Arthroplasty. [Internet]. 2012. [acesso em: 01 set 2015]; 27(2): 55-60. Disponível em: https://www.ncbi.nlm.nih.gov/pubmed/22608685.

29. Chiang CC, Chiu FY. Cefuroxime-impregnated cement and systemic cefazolin for 1 week in primary total knee arthroplasty: An evaluation of 2700 knees. Chin Med J. [Internet]. 2012. [acesso em: 05 jul 2015]; 75: 167-70. Disponível em: https://www.ncbi.nlm.nih.gov/pubmed/22541145.

30. Sewick A, Makani A, Wu C, O'Donnell J, Baldwin KD, Lee GC. Does Dual Anti-biotic Prophylaxis Better Prevent Surgical Site Infections in Total Joint Arthroplasty? Clin Orthop Relat Res. [Internet]. 2012. [acesso em: 05 set 2015]; 470(10): 2702-07. Disponível em: https://www.ncbi.nlm.nih.gov/ pubmed/22290130.

31. Zuckerman SL, Parikh R, Moore DC, Talbot TR. An evaluation of immediate-use steam sterilization practices in adult knee and hip arthroplasty procedures. Am J Infec Con-trol. [Internet]. 2012. [acesso em: 06 set 2015]; 40: 866-71. Disponível em: https://www.ncbi.nlm.nih.gov/pubmed/22364918.
32. Lenza M, Ferraz SB, Viola DCM, Filho RJG, Neto MC, Ferreti M. Epidemiologia da artroplastia total de quadril e de joelho: estudo transversal. Einstein. [Internet]. 2013. [acesso em: 23 jul 2015]; 2(11): 197-202. Disponível em: http://www.scielo. br/scielo.php?pid=S1679-45082013000200011\&script=sci_ arttext\&tlng=pt.

33. Kapadia BH, Issa K, McElroy MJ, Pivec R, Daley JÁ, Mont MA. Advance pre-operative chlorhexidine preparation reduces periprosthetic infections following total joint arthroplasty. Semin Arthroplasty. [Internet]. 2013. [acesso em: 22 jun 2015]; 2 4: 83-86. Disponível em: http://www.semarthroplasty.com/ article/S1045-4527(13)00073-4/fulltext.

34. Jonsson EO, Johannesdottir $H$, Robertsson O, Mogensen B. Bacterial contamination of the wound during primary total hip and knee replacement. Median 13 years of follow-up of 90 replacements. Acta Orthop. [Internet]. 2014. [acesso em: 12 set 2015]; 2(85): 159-64. Disponível em: https://www.ncbi. nlm.nih.gov/pmc/articles/PMC3967258/.

35. Qadir R, Sidhu S, Ochsner JL, Meyer MS, Chimento GF. Risk Stratified Usage of Antibiotic-Loaded Bone Cement for Primary Total Knee Arthroplasty: Short Term Infec-tion Outcomes With a Standardized Cement Protocol. J Arthroplasty. [Internet]. 2014. [acesso em: 05 set 2015]; 29:1622-24. Disponível em: https://www.ncbi.nlm.nih.gov/pubmed/24703363.

36. Moroski NM, Woolwine S, Schwarzkopf R. Is Preoperative Staphylococcal Decol-onization Efficient in Total Joint Arthroplasty. J Arthroplasty. [Internet]. 2015. [acesso em: 25 ago 2015]; 30: 444-46. Disponível em: https://www.ncbi.nlm. nih.gov/pubmed/25453634.

37. Peel TN, Cheng AC, Buising KL, Dowsey MM, Choong PFM. Alcoholic Chlorhex-idine or Alcoholic lodine Skin Antisepsis (ACAISA): protocol for cluster randomised con-trolled trial of surgical skin preparation for the prevention of superficial wound complica-tions in prosthetic hip and knee replacement surgery. BMJ Open. [Internet]. 2014. [acesso em: 08 set 2015]; 4: 1-6. Disponível em: https://www.ncbi.nlm.nih.gov/pmc/ articles/PMC4025412/.

38. Durando $\mathrm{P}$, et al. Adherence to international and national recommendations for the prevention of surgical site infections in Italy: Results from an observational prospective study in elective surgery. Am J of Infect Control. [Internet]. 2012. [acesso em: 25 jun 2015]; 40: 969-72. Disponível em: https://www.ncbi.nlm.nih.gov/labs/articles/22418605/.

39. Barlow D, Masud S, Rhee SJ, Ganapathi M, Andrews G. The effect of the creation of a ring-fenced orthopaedic Ward on length of stay for elective arthroplasty patients. Sur-geon. [Internet]. 2013; [acesso em: 17 jun 2015];11: 82-6. Disponível em: https://www.ncbi.nlm.nih.gov/pubmed/22717284.

40. Jonas SC, Smith HK, Blair PS, Dacombe P, Weale AE. Factors influencing length of stay following primary total knee replacement in a UK specialist orthopaedic centre. Knee. [Internet]. 2013. [acesso em: 17 jun 2015]; 20: 310-15. Disponível em: https://www.ncbi.nlm.nih.gov/ pubmed/22910196.

41. Kelava M, et al. Hospitalization before surgery increases risk for postoperative in-fections. J Thorac Cardiovasc Surg. [Internet]. 2014. [acesso em: 19 jun 2015]; 148(4): 1615-21. Disponível em: https://www.ncbi.nlm.nih.gov/ pubmed/25260276. 
42. Tan TW, et al. Shorter Duration of Femoral-Popliteal Bypass Is Associated with Decreased Surgical Site Infection and Shorter Hospital Length of Stay. J Am Coll Surg. [Internet]. 2012. [acesso em: 21 jun 2015] Oct; 215(4): 512-18. Disponível em: https://www.ncbi.nlm.nih.gov/pubmed/22819641.

43. Birgand G, et al. Air contamination for predicting wound contamination in clean surgery: A large multicenter study. Am J Infect Control. [Internet]. [acesso em: 01 jul 2015]; 1-6. Disponível em: https://www.ncbi.nlm.nih.gov/ pubmed/25752955.

44. Engesaeter LB, Lie AS, Espehaug B, Furnes O, Vollset SE, Havellin LI. Antibiotic prophylaxis in total hip arthroplasty. Effects of antibiotic prophylaxis systemically and in bone cement on the revision rate of 22,170 primary hip replacements followed 0-14 years in the Norwegian Arthroplasty Register. Acta Orthop Scand. [Internet]. 2003. [acesso em: 23 jul 2015]; 74(6): 644-51. Disponível em: https://www.ncbi.nlm.nih.gov/ pubmed/14763692.

45. Namba RS, Chen Y, Paxton EW, Slipchenko T, Fithian DC. Outcomes of Routine Use of Antibiotic-Loaded Cement in Primary Total Knee Arthroplasty. J Arthroplasty. [In-ternet]. 2009. [acesso em: 30 jun 2015]; 24: 44-47. Disponível em: https://www.ncbi.nlm.nih.gov/pubmed/19577881.

46. Chauveaux D. Preventing surgical-site infections: Measures other than antibiotics. Orthop Traumatol Surg Res. [Internet]. 2015. [acesso em: 01 jul 2015];101: 77-83. Dispo-nível em: https://www.ncbi.nlm.nih.gov/pubmed/25623269.

47. Najjar PA, Smink DS. Prophylactic Antibiotics and Prevention of Surgical Site In-fections. Surg Clin N Am. [Internet]. 2015. [acesso em: 17 jun 2015]; 95: 269-83. Disponí-vel em: https:// www.ncbi.nlm.nih.gov/pubmed/25814106. 